\title{
El comportamiento de la industria manufacturera de México ante la recesión económica de EUA
}

\author{
Jorge Eduardo Mendoza Cota \\ Investigador del Departamento de Estudios Económicos \\ de El Colegio de la Frontera Norte \\ Investigador Nacional Nivel III, Tel. 664-6316316 \\ emendoza@colef.mx
}




\section{RESUMEN}

El artículo analiza el impacto de la recesión del sector manufacturero de EUA en la industria manufacturera al nivel de subsectores y de regiones de la economía mexicana. La metodología de análisis consiste en la utilización del filtro Hodrick-Prescott para obtener indicadores del componente cíclico de las industrias manufactureras y en pruebas de estacionariedad y de equilibrio de largo plazo de las series de las manufacturas de EUA y México. Los resultados muestran una relación procíclica y volátil de las manufacturas mexicanas con las de EUA en el largo plazo.

Palabras clave: manufacturas, ciclo económico, integración económica, series de tiempo, economía mexicana

Clasificación JEL: F14, F15, E32

\section{ABSTRACT}

This paper analyzes the effect of the US manufacturing sector on the manufacturing industry of Mexico at the subsector and regional level. The methodology of analysis consists of the application of the Hodrick-Prescott filter to obtain the cyclical component for the manufacturing industries and using stationary and cointegration tests to evaluate the equilibrium of the series in the long run. The results showed a pro-cyclical and volatile relationship of the Mexican manufacturing sector with that of the USA.

Keywords: manufacturing, business cycle, economic integration, time series, the Mexican economy

JEL Clasification: F14, F15, E32

Revista de Economía, vol. XXVII, núm. 75, segundo semestre de 2010. 


\section{INTRODUCCIÓN}

La economía mundial ha venido experimentando una recesión económica y una crisis del modelo neoliberal instrumentado desde la década de los ochenta. En particular, la recesión económica de EUA ha afectado significativamente el crecimiento de los sectores económicos de México que están directamente integrados a la dinámica de la economía de EUA, como es el caso del sector manufacturero.

En efecto, un importante motor del crecimiento reciente de la economía mexicana ha estado relacionado con el dinamismo de la industria manufacturera derivado de la integración económica con Estados Unidos de América (EUA). Con base en la inversión extranjera directa (IED) y la expansión de las exportaciones manufactureras hacia EUA, las industrias mexicanas mostraron un crecimiento dinámico durante la década de los noventa (Mendoza, 2002). La capacidad de exportación de las manufacturas mexicanas estuvo relacionada con la cercanía geográfica al mercado estadounidense y con las ventajas en costos de salarios, lo que estimuló la inversión y el comercio entre México y Estados Unidos y, como resultado, profundizó la sincronización del ciclo económico ente ambas economías. Sin embargo, la industria manufacturera mostró bajos niveles de integración de cadenas productivas y, como consecuencia, alto nivel del coeficiente de importaciones (Dussel, 2009).

Desde el año de 2007 la economía de Estados Unidos (EUA) entró en un proceso de estancamiento económico que derivó en una profunda recesión económica en ese país. La drástica caída de la economía norteamericana se ha caracterizado por una profunda crisis financiera y del mercado inmobiliario, pero también por una notable desaceleración del crecimiento del sector manufacturero de ese país que, como resultado de su estrecha relación con la industria manufacturera de México, ha venido arrastrando a este sector productivo.

De esta manera, el sector manufacturero de México fue impactado negativamente en su crecimiento por la dependencia de la demanda externa de bienes manufacturados de EUA (Acevedo, 2002), por lo que ha venido experimentando severas caídas de la producción y del empleo de las ramas manufactureras que fueron claves en el sector por su rápida expansión en la década de los noventa. Es por ello, que resulta importante conocer 
cuáles son los impactos que esta desaceleración del sector manufacturero de EUA tendrá en la estructura regional y sectorial de las manufacturas mexicanas y, además, determinar cuál es el grado de sincronización de las manufacturas de México al ciclo del sector manufacturero de EUA.

En este sentido, en el presente trabajo se analizan las características de la recesión manufacturera en México y se estima el grado de sincronización del comportamiento de las manufacturas de México con las de EUA. La metodología de análisis consiste en utilizar indicadores estadísticos de correlación, autocorrelación y de las relaciones de comportamiento de las series de tiempo en el largo plazo. Lo anterior, con el objetivo de vincular el comportamiento cíclico de las manufacturas de EUA con las manufacturas de México, al nivel de subsector y al nivel estatal.

\section{LA INTEGRACIÓN MÉXICO-EUA Y EL SECTOR MANUFACTURERO}

Desde la firma del tratado de libre comercio de América del Norte (TLCAN) y la IED en México tuvieron un acelerado crecimiento, particularmente la proveniente de EUA. Dicha Inversión estuvo canalizada en particular hacia el sector de manufacturas. El crecimiento del sector manufacturero en el periodo 1996-2005 estuvo íntimamente ligado a las exportaciones de bienes intermedios y finales del sector manufacturero (y maquilador). De acuerdo con Amoroso et al. (2008), el patrón de especialización manufacturera se relacionó con la abundancia de mano de obra menos calificada en México que, conjuntamente con la cercanía geográfica, determinó una tendencia a que las empresas manufactureras se aglomeraran en la región fronteriza de México. No obstante, de acuerdo con los autores, la diferencia en las productividad y la dotación de capital humano determinó que al iniciar la década del 2000, las exportaciones, particularmente del sector maquilador, experimentaran una pérdida de mercado en la economía estadounidense en favor de países como China (Rocha, 2003) y (Mendoza, 2009).

Aunque el acelerado crecimiento de las exportaciones mexicanas llevó a México a expandir su comercio exterior durante la década de los noventa, las exportaciones manufactureras mexicanas no lograron diversificarse geográficamente, concentrándose fundamentalmente al mercado de 
EUA, al aprovechar las condiciones propicias generadas por el establecimiento del TLCAN (Guzmán y Toledo, 2005). Lo anterior determinó una dependencia de la economía mexicana en el comportamiento del mercado estadounidense y llevó a una competencia de otros países por penetrar dicho mercado.

De esta manera, a partir de la firma del TLCAN, la participación de las exportaciones mexicanas en el total de las exportaciones se elevó de un promedio de 82.6\% en 1993 a un máximo de 88.7\% en el 2000, año desde el cual se redujo su participación hasta alcanzar un promedio de $81.1 \%$ en el primer semestre de 2009, probablemente como resultado de la fuerte caída de la demanda de exportaciones mexicanas de EUA. ${ }^{1}$

Así mismo, deben destacarse que los sectores que dinamizaron las exportaciones de manufacturas fueron, en primer término, la industria de productos metálicos, maquinaria y equipo, la que participaba en promedio en 1993 con 68.8\% del total de las exportaciones manufactureras, incluyendo la actividad maquiladora, elevándose su porcentaje a $71.6 \%$ en el primer trimestre de 2009. Al interior de este subsector las ramas de transporte y comunicaciones y productos automotores mostraron una elevada participación de 24.5 y $24.7 \%$, respectivamente, en 1993, reduciéndose su participación en 2009 a 40.5 y $17.7 \%$. El otro sector de importancia es de producción de aparatos eléctricos y electrónicos que representó 36.02\% de las exportaciones manufactureras en 1993 y 31.2\% en el primer semestre de 2009. ${ }^{2}$

Es importante destacar que la liberalización económica trajo como consecuencia una expansión de la producción y exportaciones manufactureras. No obstante, diversos autores han señalado que existen importantes limitaciones del modelo de crecimiento manufacturero seguido por México. Por una parte, se aprecian restricciones externas que se derivan del desequilibrio de la balanza comercial manufacturera y, además, se observa un bajo crecimiento de la inversión bruta fija (Moreno Brid, 1999). Por otra parte, también se ha subrayado que existen problemas

\footnotetext{
${ }^{1}$ Estimaciones propias con datos del Banco de Información Económica (BIE) del INEGI.

${ }^{2}$ Estimaciones propias con datos del grupo de trabajo integrado por INEGI-BANXICO-SAT (Servicio de Administración Tributaria) y la Secretaría de Economía, publicados en el Banco de Información Económica (BIE), del Instituto Nacional de Geografía e Informática (INEGI).
} 
de disparidad del crecimiento manufacturero al nivel subsectorial y concentración de las exportaciones en pocas actividades manufactureras y alto nivel del coeficiente de importaciones del sector manufacturero (De María, Domínguez, Brown y Sánchez, 2009).

Así mismo, una gran parte del dinamismo del sector manufacturero quedó determinado por los flujos de inversión extranjera directa canalizados hacia ese sector. Este flujo de recursos de capital y tecnología se convirtió, de hecho, en otro mecanismo para profundizar la integración del ciclo de las manufacturas mexicanas al comportamiento del sector manufacturero de los EUA. Respecto a la tendencia de los flujos de IED en el sector de manufacturas se destaca también que éste tuvo una rápida expansión durante la década de los noventa llegando a representar 66.1\% de la inversión extranjera directa total en el año de 1999 (Cuadro 1).

No obstante, a partir del año 2000 se aprecia un declive de la participación de los flujos de IED en el sector manufacturero de la economía mexicana en el total de captación de IED, cayendo 55.3\% en el 2000. Esta situación se deterioró aún más como resultado de la recesión internacional, y en 2001 y 2002 se experimentaron caídas de esa participación hasta llegar a 19.8\% y 37.1\%, respectivamente. Finalmente, se destaca que después de un repunte en 2004, el porcentaje de la IED en el sector manufacturero volvió a caer en 2007 y 2008, además de presentarse este fenómeno en un contexto de una caída general de la IED total en México. Finalmente, en lo relacionado al origen de la IED, se observa que EUA sigue siendo la fuente más importante de esos flujos financieros, aunque es conveniente destacar que la proporción de la inversión extranjera directa de EUA respecto al total de la IED se ha reducido considerablemente entre el año de 2001, en que presentó el mayor porcentaje respecto al total (71.2\%), y 2008 en que cayó su participación sensiblemente $(45 \cdot 7 \%){ }^{3}$

\footnotetext{
${ }^{3}$ Estimaciones propias con datos del grupo de trabajo integrado por INEGI-BANXICO-SAT (Servicio de Administración Tributaria) y la Secretaría de Economía, publicados en el Banco de Información Económica (BIE), del Instituto nacional de Geografía e Informática (INEGI).
} 


\section{INTEGRACIÓN Y COMPONENTE CÍCLICO DE LAS MANUFACTURAS DE EUA Y DE MÉXICO}

El análisis de la sincronización de los ciclos económicos entre países generalmente utiliza indicadores del PIB o de la producción industrial (Mejía-Reyes $\mathrm{P}$, et al. 2006). Cabe destacar que el efecto que tiene una mayor integración en la sincronización del ciclo económico o industrial entre países depende tanto de la apertura económica y especialización de la producción de países, como de la especialización de la producción interindustrial y los impactos específicos por industria. En ese sentido, Krugman (1993) señala que la integración pudiera llevar a la especialización regional intraindustrial, lo que a su vez podrían derivar en diferentes niveles de sincronización del ciclo entre países, debido a la predominancia de shocks específicos por sector económico.

Adicionalmente, existe la posibilidad de que una mayor integración económica afecte la sincronización del ciclo económico, al crear shocks específicos entre países, como resultado de los impactos en la demanda agregada. Por ejemplo, el incremento del ingreso de un país puede afectar la demanda de exportaciones de otro país (Frankel y Rose, 1998). Otro instrumento de integración se relaciona con la integración comercial que puede convertirse en un mecanismo para incrementar la inversión extranjera directa, y la difusión de tecnología, generando mayor integración económica (Coe y Helpman, 1995).

Debido a que la producción y exportación de manufacturas juega un papel central en las relaciones económicas entre México y EUA, en este trabajo se estiman las características del componente cíclico del índice de crecimiento de la producción manufacturera de EUA y los índices de crecimiento de las manufacturas mexicanas al nivel total y por subsectores. Esto con la finalidad de conocer cuáles han sido las actividades manufactureras que están mas integradas al ciclo de las manufacturas norteamericanas, se evalúa el impacto que la recesión en este sector productivo de EUA ha tenido en las manufacturas de México. Se utilizan los datos de la serie de crecimiento manufacturero de acuerdo con la clasificación SCIAN (Sistema de Clasificación Industrial de América del Norte) que se obtuvieron del U.S. Department of Commerce: Bureau of Economic Análisis y las series de los subsectores manufactureros de México que se adquirieron de 
las series de la Encuesta Industrial Mensual que se encuentran en el Banco de Información Económica (BIE) del Instituto Nacional de Estadística, Geografía e Informática (INEGI).

\subsection{Integración de las manufacturas de EUA y México en el lar- go plazo}

El ciclo económico de México se ha venido vinculado cada vez más al de EUA, generando una alta dependencia de la economía mexicana respecto del comportamiento de la economía de EUA (Mendoza, 2008). Como se señaló, uno de los principales canales de trasmisión de las oscilaciones económicas está relacionado con las exportaciones manufactureras de México. Con la finalidad de realizar estimaciones de las características del grado de integración del componente cíclico de las series de tiempo de las manufacturas analizadas es importante establecer, en primer término, si las series consideradas individualmente son estacionarias a lo largo del tiempo, ya que de no serlo pueden arrojar estimaciones espurias. Con este propósito se requiere determinar si las series exhiben vectores de cointegración, para lo cual se realizan las pruebas Dickey-Fuller aumentada (DFA) y la prueba de Phillips-Perron.

La prueba Dickey y Fuller aumentada (DFA) es el método más utilizado para hacer la prueba de raíces unitarias. Las siguientes ecuaciones para determinar la presencia o no de raíces unitarias:

$$
\begin{aligned}
& \Delta Y_{t}=\delta Y_{t-1}+\mathrm{v}_{t} \\
& \Delta Y_{t}=\alpha+\beta T+\delta Y_{t-1}+\gamma \sum_{i=1}^{\rho} T_{t-1}+\mathrm{v}_{t} \\
& \Delta Y_{t}=\alpha+\delta Y_{t-1}+\mathrm{v}_{t} \\
& \Delta Y_{t}=\alpha+\beta T+\delta Y_{t-1}+\gamma \sum_{i=1}^{\rho} T_{t-1}+\mathrm{v}_{t} \\
& \Delta Y_{t}=\alpha+\beta T+\delta Y_{t-1}+\gamma \sum_{i=1}^{\rho} T_{t-1}+\mathrm{v}_{t}
\end{aligned}
$$


Donde el término $\delta$ es el parámetro autoregresivo, $\gamma$ es la tendencia en el tiempo y $v_{t}$ es la perturbación. Las diferencias entre estas cuatro regresiones determina la presencia de un componente aleatorio y de componentes determinísticos como el intercepto y la tendencia.

Así mismo, en la última regresión se incorpora un componente de retardo para asegurar que los retardos sean ruido blanco (series estacionarias sin diferenciar). El parámetro que es relevante para realizar la prueba es $\delta$. Cuando $\delta=$ o la hipótesis nula es de no estacionariedad cuando $\delta \neq$ o, la hipótesis es de estacionariedad.

En el Cuadro 2 se presentan las pruebas de raíces unitarias que se realizan comparando el valor $t$ (tau) con los valores críticos de MacKinnon. Los resultados muestran que la mayoría de las industrias manufactureras de México, en el periodo analizado, exhiben valores de los estadísticos tau superiores a los valores críticos, por lo que se puede considerar que las series son estacionarias. Inicialmente se estimó la prueba Dickey-fuller para las series transformadas en logaritmos, y los resultados mostraron una aceptación de la hipótesis nula de no estacionariedad, por lo que se transformaron dichas series en primeras diferencias. Se realizaron nuevas estimaciones de la prueba con las series de tiempo en primeras diferencias donde se obtuvo como resultado que la mayoría de los subsectores manufactureros resultaron con series estacionarias. La excepción fueron los casos de las industrias textiles excepto prendas de vestir, las de plástico y hule y las industrias de productos minerales no metálicos y productos metálicos. Así pues, 17 subsectores manufactureros mostraron que las series son estacionarias e integradas de orden uno I(1), por lo que se pueden realizar estimaciones de largo plazo con esas series de manera individual, lo que nos permite tener mayor confianza en los resultados de las estimaciones del componente cíclico de las series.

A fin de corroborar si las industrias manufactureras que fueron no estacionarias bajo la prueba de Dickey-Fuller aumentado arrojan el mismo resultado bajo una prueba no paramétrica se estimó la prueba PhillipsPerron (1988). Dicha prueba de raíces unitarias controla la existencia de autocorrelación serial, al modificar la razón de coeficiente de la prueba DFA para que la autocorrelación no afecte la distribución asintótica de la prueba. La prueba PP mostró mejores resultados, ya que todas las series de los subsectores manufactureros mostraron no tener raíces unitarias 
cuando se tomó la primera diferencia de las series, por lo que esta prueba viene a corroborar la prueba DFA, sugiriendo que las series de los subsectores manufactureros son estacionarias a lo largo del tiempo.

Otro aspecto importante que debe verificarse antes de realizar las pruebas de correlación entre los índices de crecimiento manufacturero de México y de EUA se relaciona con las pruebas de cointegración de las series de ambos países. En particular, ya que las series de tiempo de las manufacturas de EUA y de los subsectores manufactureros de México son estacionarias, buscamos determinar si la combinación de las series de EUA y de México cointegran en el tiempo, es decir, si existe alguna combinación lineal de las series genera una relación estacionaria. Si existe esta condición, entonces podremos estar seguros que cualquier correlación de largo plazo entre las series del índice de crecimiento de la manufacturas de EUA y de los subsectores manufacturas de México no será espuria.

De esta manera, sí se cumplen las condiciones de estacionariedad de todas las series individuales al tomarles la primera diferencia y de estacionariedad de al menos una combinación de las series de México con las de EUA. Esto equivale a corroborar la existencia de una relación económica de equilibrio de largo plazo entre las series de tiempo que están integradas de orden I (1), como es el caso o de las series mencionadas.

Para corroborar las relaciones de largo plazo de las variables se realizaron estimaciones de la prueba de Johansen. La utilidad de la prueba es que nos permite conocer el número de vectores de cointegración con sus respectivas velocidades de ajuste, determinadas por los valores de los coeficientes de las variables que cointegran; el procedimiento analiza la significancia de las raíces características (eigenvalues) y la prueba de traza. La hipótesis nula de ambas pruebas es la no existencia de vectores de cointegración.

Los resultados de la prueba de cointegración de Johansen mostraron que del total de las series de los subsectores manufactureros de México y del sector manufacturero de EUA arrojaron valores críticos que determinan que, al menos, existió un vector de cointegración entre cada uno de los subsectores manufactureros de México y la serie del sector manufacturero de EUA (Cuadro 3). Por ello, se puede señalar que las estimaciones muestran la existencia de una relación de equilibrio de largo plazo en la 
industria manufacturera mexicana al nivel de subsectores y la industria manufacturera de EUA en su conjunto. Lo anterior nos permite sugerir que los resultados de las mediciones del componente cíclico corresponden a un nivel de equilibrio de las series estimadas.

\subsection{Componente cíclico de las manufacturas por subsectores y estados}

Con el fin de estimar la tendencia y el componente cíclico de las series utilizadas se calcula el filtro Hodrick-Prescott (Hodrick y Prescott, 1981), el cual suaviza las series económicas y permite establecer la metodología para determinar la tendencia y componente cíclico económico. El objetivo fundamental es analizar el grado de sincronización de la industria manufacturera mexicana al ciclo manufacturero de EUA utilizando los índices de crecimiento de la producción manufacturera en EUA y los índices de crecimiento del empleo manufacturero por subsectores de acuerdo al SCIAN. ${ }^{4}$ Con base en esta información es posible analizar la relación de los componentes cíclicos de las series de ambos países ${ }^{5}$ utilizando diversas estimaciones estadísticas como los coeficientes de correlación, de autocorrelación, las desviaciones estándar y las pruebas de cointegración del PIB de México y de EUA.

Los resultados de las estimaciones del componente cíclico de las manufacturas mexicanas por subsectores muestran, en primer término, que la industria en su conjunto muestra una tendencia procíclica con respecto al componente de EUA al exhibir un coeficiente de correlación de o.823. Así mismo, se destaca que los subsectores con una correlación procíclica mayor son los siguientes: la industria de plástico y hule, la industria de productos minerales no metálicos, las industrias metálicas, los productos metálicos y la industrias de equipo de transporte (Cuadro 4). Otras

\footnotetext{
${ }^{4}$ Sistema de Clasificación Industrial de América del Norte.

${ }^{5} \mathrm{El}$ filtro HP divide a una serie de tiempo en la suma de un componente de crecimiento $\mathrm{y}_{\mathrm{t}} \mathrm{y}$ un componente cíclico $c_{t}$. Representándose de la siguiente manera: yt $=$ gt + ct La solución del componente de crecimiento se presenta de la siguiente manera: $\operatorname{Min}\left\{\sum \mathrm{t}=1 \mathrm{ct}{ }^{\mathrm{t}}+\lambda \sum \mathrm{t}=1\left[\left(\mathrm{~g}_{\mathrm{t}}-\mathrm{g}_{\mathrm{t}-1}{ }^{\mathrm{t}}\right)-\left(\mathrm{g}_{\mathrm{t}-1}-\mathrm{g}_{\mathrm{t}-2}\right)\right]^{2}\right\}$ donde los componentes cíclicos son las desviaciones del sendero de crecimiento y la suavización del componente de crecimiento es medido como la suma de los cuadrados de su segunda diferencia. El parámetro $\lambda$ es un número positivo que penaliza la variabilidad del componente del crecimiento, mientras más largo es más suavizado es $\mathrm{g}_{\mathrm{t}}$.
} 
industrias que se correlacionaron positivamente con el componente cíclico de EUA son las de maquinaria y equipo, las industrias de madera y papel y la fabricación de muebles.

En lo relacionado con las repercusiones del impacto de los cambios en las tendencias del componente cíclico de las series de tiempo de los subsectores de las manufacturas mexicanas, se aprecia que existe una mayor volatilidad del componente cíclico de la industria de fabricación de equipo de transporte, de equipo de computación y componentes electrónicos de maquinaria y equipo y de la industria de la madera, ya que las desviaciones estándar del componente cíclico de estas series fueron muy superiores al del componente cíclico de EUA, lo que determinó que el indicador de volatilidad fuera superior a la unidad en todos los casos mencionados. Estos resultados indican que los subsectores más dinámicos de la industria manufacturera mexicana exhiben un componente cíclico mucho más volátil, lo que determina fases recesivas más profundas y frecuentes que su contraparte manufacturera de EUA.

En relación con el efecto de los cambios del componente cíclico de las manufacturas de EUA al nivel de las manufacturas estatales de la economía mexicanas, en el Cuadro 7 se presentan los coeficientes de correlación de las manufacturas de EUA y de las manufacturas mexicanas, apreciándose que las estimaciones de la correlación de los componentes cíclicos estatales sólo muestran un impacto procíclico considerable en los estados de Baja California, Nuevo León, Coahuila, Querétaro, Michoacán, Tamaulipas y Chiapas (Cuadro 5). Al respecto, se puede señalar que una proporción importante de los estados con coeficientes de correlación procíclicos se localizaron en la frontera norte del país, lo que tiende a corroborar que el efecto de la recesión de la economía de EUA ha generado un impacto negativo mayor en la región de la frontera norte de México.

No obstante, la persistencia de los cambios cíclicos es generalizada al nivel estatal. Además, se destaca que estados como Chihuahua, Coahuila, Aguascalientes, Guanajuato, Sonora, Chiapas y Tabasco mostraron elevados índices de volatilidad del componente cíclico, lo que implica que en varios estados de la frontera norte las oscilaciones cíclicas tienden a ser mucho más prolongadas. Lo anterior nos muestra evidencia de que no solamente los estados de la frontera y algunos estados con producción manufacturera importante como Querétaro y Guanajuato se relacionan 
con el componente cíclico de EUA, sino que además estos estados sufren de manera más prolongada las etapas recesivas del ciclo.

\section{LA RECESIÓN ECONÓMICA DE EUA Y LAS MANUFACTURAS DE MÉXICO}

Uno de los sectores económicos que ha experimentado con mayor fuerza la tendencia recesiva en la economía de EUA ha sido el sector manufacturero. En el periodo de enero de 2008 a abril de 2009 el sector manufacturero de ese país cayó $16.5 \%$, mientras que el índice de crecimiento del empleo manufacturero en ese periodo en México cayó 9.4\%. ${ }^{6}$ Se observa que el alto grado de sincronización del ciclo económico de estos sectores en ambos países ha determinado un alto grado de dependencia del comportamiento del sector manufactureras de México con respecto a la evolución de la producción manufacturera en EUA (Gráfica 1).

No obstante, se aprecia que las manufacturas mexicanas en el periodo señalado mostraron una tendencia de crecimiento moderado con respecto a las de EUA, mostrando un desaceleramiento más suavizado que el que se ha venido presentado en el caso de EUA. Lo anterior, como resultado de la pérdida de dinamismo del crecimiento de importantes industrias manufactureras (principalmente maquiladoras) en México, a partir de la recesión económica experimentada en el 2001 y como consecuencia de la creciente competencia de las manufacturas de China en el mercado norteamericano.

De esta manera, aunque las manufacturas de México en su conjunto parecen haber entrado en una caída relativamente menos drástica que la de EUA, al analizar los diferentes subsectores que componen la industria manufacturera se constata que algunas industrias se han visto afectadas negativamente, en particular, las íntimamente ligadas al crecimiento de la industria manufacturera norteamericana, como proveedoras directas de insumos a empresas manufactureras de este país y las que están relacionadas al mercado de consumidores de bienes finales en EUA.

\footnotetext{
${ }^{6}$ De acuerdo con estimaciones propias con base en datos de la Federal Reserve en Estados Unidos y de la Encuesta Industrial Mensual de México.
} 


\subsection{Los principales sectores manufactureros afectados por la recesión económica}

$\mathrm{Al}$ analizar el comportamiento de la industria manufacturera de México al nivel de subsectores ante el impacto de la recesión internacional, se aprecia que si bien el conjunto del sector manufacturero ha respondido con una reducción generalizada de la actividad productiva han sido, con excepción de algunas industrias orientadas al mercado interno como la de alimentos, las relacionadas con las exportaciones al mercado de EUA las que han experimentado drásticas caídas en sus tendencias de crecimiento.

En el Cuadro 6 se presentan los cinco subsectores manufactureros con mayores tasas negativas de crecimiento porcentual acumulado entre el 2005 y abril de 2009. De esta información se destaca que las ramas incluidas en el subsector de equipo de transporte fueron las que se han visto más adversamente afectadas por la recesión internacional. Así mismo dicho subsector, en el periodo analizado, tuvo una contracción porcentual acumulada del $\mathbf{- 2 0 . 4 \%}$, presentando un estancamiento en el crecimiento en el 2007 del $0.1 \%$, seguido de una severa caída del -13.6 en año del 2008 , acumulando una caída adicional del $6.8 \%$ hasta el cuarto trimestre de 2009. Por ello, es posible concluir que este sector que incluye las industrias productoras de automóviles y camionetas y equipo, de transporte y autopartes, ha sido el más afectado por la recesión internacional, particularmente la que se ha experimentado en la economía de EUA. Lo anterior pone en evidencia que las ramas manufactureras que tendieron a mostrar mayores tasas negativas de crecimiento estuvieron relacionadas con una mayor integración al comportamiento de la economía norteamericana y su efecto en la demanda de importaciones de bienes manufacturados provenientes de México.

El siguiente subsector que entró en una severa recesión es el de la fabricación de computadoras, equipos de comunicación y electrónicos que cayó un $12.5 \%$ en términos acumulados en el periodo referido. Se destaca también que este sector venía mostrando ya los efectos del estancamiento en el crecimiento derivados del efecto de la crisis de $2001 \mathrm{y}$ del impacto de las exportaciones de China al mercado norteamericano. De esta forma, las exportaciones de insumos electrónicos y componentes de computadoras también sufrieron el efecto de la reducción de la demanda de las empresas 
y consumidores de EUA. Además, estas industrias también venían mostrando un estancamiento desde 2005 que se convirtió en un franco crecimiento negativo en el año de 2008.

Otros subsectores manufactureros que también se vieron afectados fueron la producción de muebles, de textiles y de prendas de vestir, todos ellos con tasas de crecimiento negativas en 2008 por arriba de 7\%. Las actividades manufactureras que experimentaron la recesión económica incidieron en la tendencia negativa del sector manufacturero en su conjunto y evidenciaron la dependencia de las ramas manufactureras más dinámicas con respecto del mercado de EUA. Lo anterior refleja, por tanto, el resultado del proceso de integración del ciclo de la producción manufacturera de EUA con las principales ramas manufactureras exportadoras de la economía mexicana.

Con respecto a la recesión del sector manufacturero mexicano al nivel regional, se aprecia que los estados con crecimientos negativos más pronunciados fueron los que se localizaron en la frontera norte de México o en estados con alta vocación manufacturera. En particular, se experimentaron drásticas caídas del crecimiento manufacturero en los estados de Coahuila, Chihuahua y Baja California que se caracterizan por contar con una importante industria automotriz, siendo esta actividad una de las más afectadas tanto por la caída de la producción automotriz en EUA como por la reducción de la demanda en el mercado estadounidense de automóviles (Cuadro 7).

De esta manera, la caída porcentual del crecimiento acumulado entre enero de 2008 y febrero de 2009 de las manufacturas en los estados de Coahuila y Chihuahua fue de -34.3 y -24.0\%, respectivamente. Así mismo, se destaca el caso de Baja California que también exhibió una caída pronunciada de $20.5 \%$, como resultado de la salida de importantes empresas maquiladoras de esa región, en particular las relacionadas con la industria electrónica.

Por su parte, los estados que mostraron tasas de crecimiento porcentual mensual acumuladas positivas fueron Veracruz, Campeche, Nayarit y Oaxaca. Por tanto, es posible concluir que los estados de la frontera norte o los estados con mayor vocación manufacturera son los que han sido más afectados por la recesión del sector manufacturero de EUA, mientras que los estados del sur o con baja participación de 
la producción manufacturera, particularmente la de exportación han resentido con menor fuerza el impacto de la recesión de EUA, debido a las menor sincronización de la producción manufacturera en ambos países.

\section{CONCLUSIONES}

La crisis económica de EUA de 2008 ha mostrado problemas estructurales para generar crecimiento sostenido tanto en el sector financiero como en la producción manufacturera. El estancamiento y declive de la economía internacional ha descubierto las limitaciones del modelo de crecimiento adoptado por la economía mexicana, particularmente en lo relacionado a la alta dependencia de los sectores externo y manufacturero respecto de la dinámica de crecimiento de la economía estadounidense.

El impacto de la recesión se ha dejado sentir particularmente en algunos sectores de la economía mexicana como es el manufacturero. El desempeño de las actividades manufactureras durante el año de 2008 fue marcadamente negativo y está directamente relacionado con la alta dependencia del comportamiento del mercado estadounidense y los flujos de IED provenientes de ese país, lo que ha determinado un alto nivel de integración económica en ese sector y un comportamiento del componente cíclico de las manufacturas mexicanas altamente relacionado al de las manufacturas de EUA.

De esta manera, el alto grado de sincronización del ciclo de la industria manufacturera de México y de EUA y las diferencias de la industria manufacturera al nivel de los diverso subsectores y de las regiones en México han determinado, en un contexto de integración al mercado de EUA, las siguientes características de la tendencia recesiva del sector manufacturero en México:

1. Se corrobora con pruebas econométricas una relación económica de equilibrio de largo plazo entre los movimientos del índice de la producción manufacturera de EUA y los subsectores de la industria manufacturera de México. Lo anterior permite realizar estimaciones de largo plazo sobre la correlación de los componentes cíclicos de las series de tiempo de ambas industrias. 
2. Las series de tiempo manufactureras mostraron un alto nivel de correlación positiva (procíclica) entre los subsectores manufactureros de México y el sector manufacturero de EUA, por lo que se corrobora un impacto en la misma dirección del ciclo manufacturero de EUA en los subsectores manufactureros de México.

3. Los subsectores con una correlación procíclica mayor son los siguientes: la industria de plástico y hule, la industria de productos minerales no metálicos, las industrias metálicas, los productos metálicos y las industrias de equipo de transporte. Estas industrias se caracterizan por estar relacionadas directamente con las exportaciones de bienes manufacturados a EUA y han sido severamente afectadas por la recesión internacional.

4. Además, existe una mayor volatilidad del componente cíclico de la industria de fabricación de equipo de transporte, de equipo de computación y componentes electrónicos de maquinaria y equipo y de la industria de la madera, lo que implica que el impacto de la recesión de las manufacturas norteamericanas tienen un efecto que tiende a perdurar aún más que la propia recesión del sector manufacturero de EUA.

5. Las estimaciones de la correlación de los componentes cíclicos estatales muestran que la mayoría de los estados de la frontera norte tienen coeficientes de correlación procíclicos, destacándose los estados de Baja California, Nuevo León, Coahuila y Tamaulipas. Por su parte, la persistencia de los cambios cíclicos es generalizada al nivel estatal, sobresaliendo la volatilidad en los estados de Chihuahua, Coahuila, Aguascalientes, Sonora y Guanajuato, por lo que además estos estados experimentan etapas recesivas del ciclo más largas.

6. La crisis del sector no sólo afecta al país en su conjunto en términos de las exportaciones y divisas, sino que ha generado una seria recesión del sector manufacturero al nivel de la región de los principales estados manufactureros del norte del país a partir de 2008.

7. La concentración de la producción manufacturera para exportación hacia el mercado estadounidense, particularmente del subsector de equipo de transporte y de computación y componentes electrónicos, ha determinado una severa contracción del sector manufacturero en su conjunto. Asimismo la recesión del sector manufacturero se ha dejado sentir con mayor profundidad en los estados de la región fronteriza de México. 
Así pues, las perspectivas de la industria manufacturera mexicana parecen estar limitadas y dependientes tanto de la reanimación del mercado y de la producción manufacturera de EUA. En este sentido, se aprecia la existencia de una dependencia sectorial y regional de la dinámica de las manufacturas mexicanas y una perspectiva de recuperación de largo plazo lenta y que deberá de ir acompañada de una reestructuración de las estrategias de producción y exportación claramente delineadas al nivel internacional. El estancamiento, la crisis y la poca capacidad de respuesta de la industria manufacturera mexicana ante los cambios de la economía de EUA, sugieren la conveniencia de hacer una revisión de la política industrial y de apoyo al sector.

\section{BIBLIOGRAFÍA}

Acevedo Fernández, Ernesto, (2002) "Causas de la recesión de la industria maquiladora”, Momento Económico, núm. 124, pp. 11-25.

Amoroso, Nicolás, Daniel Chiquiar, Núria Quella y Manuel Ramos-Francia, (2008) "Determinantes de la ventaja comparativa de las exportaciones manufactureras mexicanas en el periodo 19962005”, Banco de México, Documentos de Investigación, $\mathrm{N}_{-}$ 2008-01.

Coe, D.T. y E. Helpman, (1995) “International R\&D spillovers”, European Economic Review, núm. 39, pp. 859-887.

De María y Campos, Mauricio, Lilia Domínguez Villalobos, Flor Brown Grossman y Armando Sánchez, (2009). "El desarrollo de la industria mexicana en su encrucijada”, Universidad Iberoamericana, Instituto de Investigaciones sobre el Desarrollo Sustentable y Equidad Social, Mimeo.

Dussel Enrique, (2009) "La manufactura mexicana: copciones de recuperación?”, Economía Informa, núm. 357, pp: 41-52.

Frankel, J.A., y A.K. Rose, (1998) "The endogeneity of the optimum currency area criteria”, The Economic Journal 108, pp. 1009-1025. 
Guzmán, Alenka y Alejandro Toledo, (2005) "Competitividad económica de México y China en el mercado estadounidense”, Economía UNAM, Vol. 2, Núm. 4, pp. 1-44.

Hodrick, R. and Prescott, E.,(1997) "Post-war U.S. Business Cycles: An Empirical Investigation” Working Paper, Carnegie-Mellon, University, 1981. Reprinted in Journal of Money, Credit and Banking, Vol. 29, No. 1, February 1997.

Krugman, Paul, (1993) Lessons of Massachusetts for EMU. En Torres, F. and F. Giavazzi (eds.) Adjustment and growth in the European Monetary Union, Oxford; New York and Melbourne: Cambridge University Press.

Mejia-Reyes et al. (2006) "Los claroscuros de la sincronización internacional de los ciclos: evidencia sobre la manufactura en México", Ciencia Ergo Sum, Vol. 13, pp: 133-142.

Mendoza Jorge Eduardo, "Agglomeration economies and urban and manufacturing growth in the northern border cities of Mexico", Economia Mexicana, Vol. XI, núm. 1. 2008, "El impacto de la integración económica en la sincronización del ciclo económico entre México y los EUA", Boletín del Sistema de Información Geográfica de INEGI, Vol 1, Núm. 1, pp. 31-45.

(2009) "Las exportaciones de China y los determinantes locales de empleo de las maquiladoras de la frontera norte de México", Región y Sociedad, Vol. XXI, pp. 117-144.

Moreno Brid, Juan Carlos, (1999) Reformas Macroeconómicas e Inversión Manufacturera en México, CEPAL, Serie Reformas Económicas.

Phillips Peter y Pierre Perron, (1998) “Testing for Unit roots in times series regression”, Biometrika, Vol. 75, núm. 2, pp. 335-346.

Rocha, Lourdes, "Competencia entre México y China en el mercado de importaciones de Estados Unidos de América”, Examen de la Situación económica de México, Vol.79. 


\section{Cuadro 1}

Evolución de la Inversión Extranjera Directa en el Sector Manufacturero de México, 1994-2008

\begin{tabular}{|c|c|c|}
\hline & $\begin{array}{c}\text { Inversión } \\
\text { Extranjera }\end{array}$ & $\begin{array}{l}\text { Inversión Extranjera Directa } \\
\text { en el sector manufacturero } \\
\text { como porcentaje del total }\end{array}$ \\
\hline Periodo & Total & de la IED \\
\hline 1994 & $\$ 10,646.9$ & $58.16 \%$ \\
\hline 1995 & $\$ 8,374.6$ & $58.06 \%$ \\
\hline 1996 & $\$ 7,847.9$ & $61.41 \%$ \\
\hline 1997 & $\$ 12,145.6$ & $60.03 \%$ \\
\hline 1998 & $\$ 8,373.5$ & $59.83 \%$ \\
\hline 1999 & $\$ 13,858.6$ & $66.11 \%$ \\
\hline 2000 & $\$ 18,019.6$ & $55.32 \%$ \\
\hline 2001 & $\$ 29,817.7$ & $19.82 \%$ \\
\hline 2002 & $\$ 23,728.9$ & $37.07 \%$ \\
\hline 2003 & $\$ 16,521.6$ & $46.91 \%$ \\
\hline 2004 & $\$ 23,681.1$ & $55.77 \%$ \\
\hline 2005 & $\$ 21,976.8$ & $50.21 \%$ \\
\hline 2006 & $\$ 19,428.0$ & $50.85 \%$ \\
\hline 2007 & $\$ 27,528.1$ & $44.28 \%$ \\
\hline 2008 & $\$ 22,481.2$ & $29.04 \%$ \\
\hline & &
\end{tabular}

Fuente: Estimaciones propias con datos elaborados por la Secretaría de Economía. Dirección General de Inversión Extranjera publicados en el Banco de Información Económica de INEGI. 
El comportamiento de la industria manufacturera de México...

Cuadro 2

\begin{tabular}{|c|c|c|c|c|c|c|c|c|c|c|}
\hline \multicolumn{11}{|c|}{ Pruebas de raíz unitaria } \\
\hline \multicolumn{11}{|c|}{$\begin{array}{l}\text { Primera diferencia de los índices manufactureros de los EUA y de los subsectores manufactureros de } \\
\text { México (SCIAN) }\end{array}$} \\
\hline \multirow[b]{2}{*}{ Sector } & \multirow[t]{2}{*}{\begin{tabular}{|c|} 
Estadístico \\
Dickey-Fuller \\
aumentado \\
\end{tabular}} & \multicolumn{3}{|c|}{ Valores críticos del test: } & \multirow[t]{2}{*}{ Prob. } & \multirow[t]{2}{*}{\begin{tabular}{|c|} 
Estadistico \\
Phillips- \\
Perron \\
\end{tabular}} & \multicolumn{3}{|c|}{ Valores críticos del test: } & \multirow[t]{2}{*}{ Prob. } \\
\hline & & Al $1 \%$ & Al $5 \%$ & Al 10\% & & & Al $1 \%$ & Al $5 \%$ & Al $10 \%$ & \\
\hline 311 Alimentos & -7.9976 & -3.5683 & -2.9212 & -2.5986 & 0.0000 & -7.9976 & -3.5683 & -2.9212 & -2.5986 & 0.0000 \\
\hline $\begin{array}{l}312 \text { Bebidas y } \\
\text { del tabaco }\end{array}$ & -7.9972 & -3.5683 & -2.9212 & -2.5986 & 0.0000 & -8.1498 & -3.5683 & \begin{tabular}{l|l|}
3 & -2.9212 \\
\end{tabular} & -2.5986 & 0.0000 \\
\hline $\begin{array}{l}313 \text { Insumos } \\
\text { textiles }\end{array}$ & -5.7861 & -3.5683 & -2.9212 & -2.5986 & 0.0000 & -5.7549 & 3.5683 & \begin{tabular}{|l|l|}
3 & -2.9212 \\
\end{tabular} & -2.5986 & 0.0000 \\
\hline \begin{tabular}{|l|}
314 Textiles, \\
excepto prendas \\
de vestir
\end{tabular} & -1.1066 & -3.5885 & -2.9297 & -2.6031 & 0.7050 & -9.1230 & -3.5683 & \begin{tabular}{|l|} 
\\
\end{tabular} & -2.5986 & 0.0000 \\
\hline \begin{tabular}{|l|}
315 Prendas de \\
vestir \\
316 Cuero y piel \\
\end{tabular} & $\begin{array}{r}-6.2526 \\
-6.0530 \\
\end{array}$ & \begin{tabular}{|l|}
-3.5683 \\
-3.5683 \\
\end{tabular} & \begin{tabular}{|l|}
-2.9212 \\
-2.9212 \\
\end{tabular} & $\begin{array}{l}-2.5986 \\
-2.5986 \\
\end{array}$ & $\begin{array}{l}0.0000 \\
0.0000\end{array}$ & $\begin{array}{l}-6.2555 \\
-6.0508 \\
\end{array}$ & \begin{tabular}{|l|}
-3.5683 \\
-3.5683 \\
\end{tabular} & \begin{tabular}{|l|l|}
3 & -2.9212 \\
3 & -2.9212 \\
\end{tabular} & \begin{tabular}{|l|}
-2.5986 \\
-2.5986 \\
\end{tabular} & $\begin{array}{l}0.0000 \\
0.0000 \\
\end{array}$ \\
\hline 321 Madera & -5.9971 & -3.5683 & -2.9212 & -2.5986 & & -6.2545 & -4.1525 & -3.5024 & -3.1807 & \\
\hline 322 Papel & -6.4425 & -3.5683 & -2.9212 & -2.5986 & 0.0000 & -6.5045 & -3.5683 & \begin{tabular}{|l|} 
\\
\end{tabular} & -2.5986 & 0.0000 \\
\hline 323 Impresión & -9.9170 & -3.5683 & -2.9212 & -2.5986 & 0.0000 & -12.6330 & -4.1525 & -3.5024 & -3.1807 & 0.0000 \\
\hline $\begin{array}{l}324 \text { Derivados } \\
\text { del petróleo y } \\
\text { del carbón }\end{array}$ & -13.6120 & -3.5683 & -2.9212 & -2.5986 & 0.0000 & -14.1355 & -3.5683 & \begin{tabular}{l|l|}
3 & -2.9212 \\
\end{tabular} & -2.5986 & 0.0000 \\
\hline 325 Química & -8.6593 & -3.5683 & -2.9212 & -2.5986 & 0.0000 & -8.6573 & -3.5683 & -2.9212 & -2.5986 & 0.0000 \\
\hline $\begin{array}{l}326 \text { Plástico y } \\
\text { del hule }\end{array}$ & 2621 & -3.5713 & -2.9225 & -2.5992 & 0.1880 & -4.6891 & -3.5683 & \begin{tabular}{|l|} 
\\
\end{tabular} & -2.5986 & 0.0004 \\
\hline $\begin{array}{l}327 \text { Productos } \\
\text { de minerales no } \\
\text { metálicos }\end{array}$ & -3.0348 & -4.1611 & -3.5064 & -3.1830 & 0.1340 & -4.3698 & -3.5683 & \begin{tabular}{|l|} 
\\
\end{tabular} & -2.5986 & 0.0010 \\
\hline $\begin{array}{l}331 \text { Metálicas } \\
\text { básicas }\end{array}$ & -3.0222 & -3.5713 & -2.9225 & -2.5992 & 0.0400 & -6.2473 & -4.1525 & -3.5024 & -3.1807 & 0.0000 \\
\hline $\begin{array}{l}332 \text { Productos } \\
\text { metálicos }\end{array}$ & -1.7731 & -3.5713 & -2.9225 & -2.5992 & 0.3890 & -4.9099 & -3.5683 & \begin{tabular}{l|l|}
3 & -2.9212 \\
\end{tabular} & -2.5986 & 0.0002 \\
\hline \begin{tabular}{l|}
333 Maquinaria \\
y equipo
\end{tabular} & -3.6158 & -3.5683 & -2.9212 & -2.5986 & 0.0090 & -3.9130 & -4.1525 & -3.5024 & -3.1807 & 0.0186 \\
\hline \begin{tabular}{|l|}
34 \\
Computación y \\
comunicación \\
\end{tabular} & -5.5184 & -3.5683 & -2.9212 & -2.5986 & 0.0000 & -5.5986 & -3.5683 & \begin{tabular}{l|l|}
3 & -2.9212 \\
\end{tabular} & -2.5986 & 0.0000 \\
\hline $\begin{array}{l}335 \text { Equipo de } \\
\text { generación } \\
\text { eléctrica }\end{array}$ & -3.6496 & -3.5683 & -2.9212 & -2.5986 & 0.0080 & -4.5708 & -4.1525 & - -3.5024 & -3.1807 & 0.0031 \\
\hline $\begin{array}{l}336 \text { Fabricación } \\
\text { de equipo de } \\
\text { transporte }\end{array}$ & -5.2243 & -4.1525 & -3.5024 & -3.1807 & 0.0005 & -2.7566 & -3.5683 & \begin{tabular}{|l|l|} 
& -2.9212 \\
\end{tabular} & -2.5986 & 0.0071 \\
\hline 337 Muebles & -5.2310 & -3.5683 & -2.9212 & -2.5986 & 0.0001 & -5.4914 & -3.5683 & -2.9212 & -2.5986 & 0.0000 \\
\hline \begin{tabular}{|l|}
339 \\
industrias
\end{tabular} & -8.0187 & -4.1525 & -3.5024 & -3.1807 & 0.0000 & -8.1018 & -3.5683 & \begin{tabular}{|l|} 
\\
\end{tabular} & -2.5986 & 0.0000 \\
\hline
\end{tabular}




\section{Cuadro 3}

\begin{tabular}{|c|c|c|c|c|c|c|c|c|c|c|}
\hline \multicolumn{11}{|c|}{$\begin{array}{c}\text { Prueba de cointegración de las series del índice manufacturero de los EUA y los principales sectores } \\
\text { manufactureros de México (SCIAN) }\end{array}$} \\
\hline \multirow[t]{2}{*}{ Sector } & \multicolumn{5}{|c|}{ Prueba de traza } & \multicolumn{5}{|c|}{ Prueba de eigenvalues } \\
\hline & No. of $\mathrm{CE}(\mathrm{s})$ & Eigenvalues & Est. & $\begin{array}{l}\text { Valor } \\
\text { crítico }\end{array}$ & Prob. ** & No. of $\mathrm{CE}(\mathrm{s})$ & Eigenvalues & Estadís. & $\begin{array}{l}\text { Valor } \\
\text { crítico }\end{array}$ & Prob.** \\
\hline \multirow[t]{2}{*}{311 Alimentos } & & 0.3546 & 26.6087 & 25.8721 & 0.0405 & & 0.3546 & $\begin{array}{ll}61.8955 \\
6\end{array}$ & $\begin{array}{ll}5 & 19.3870\end{array}$ & 0.0212 \\
\hline & Al menos $1 *$ & 0.0900 & 4.7131 & 12.5180 & 0.6379 & $\mathrm{Al}$ menos 1 * & 0.0900 & \begin{tabular}{|l}
0.7131 \\
\end{tabular} & 112.5180 & 0.6379 \\
\hline \multirow[t]{2}{*}{312 Beb. y tab. } & & 0.3453 & 25.6235 & 20.2618 & 0.0082 & & 0.3453 & \begin{tabular}{|l|l}
3 & 21.1822
\end{tabular} & 215.8921 & 0.0067 \\
\hline & Al menos $1 *$ & 0.0850 & 4.4413 & 9.1645 & 0.3504 & Al menos $1^{*}$ & 0.0850 & \begin{tabular}{l|l}
0 & 4.4413 \\
\end{tabular} & \begin{tabular}{l|l}
3 & 9.1645 \\
\end{tabular} & 0.3504 \\
\hline \multirow[t]{2}{*}{313 Insum. textiles } & & 0.3623 & 24.1930 & 15.4947 & 0.0019 & & 0.3623 & \begin{tabular}{|l|l}
3 & 22.4960 \\
\end{tabular} & 014.2646 & 0.0020 \\
\hline & Al menos 1 * & 0.0334 & 1.6971 & 3.8415 & 0.1927 & & 0.0334 & \begin{tabular}{l|l}
1.6971 \\
\end{tabular} & \begin{tabular}{l|l}
1 & 3.8415 \\
\end{tabular} & $\begin{array}{ll}5.1927 \\
\end{array}$ \\
\hline \multirow[t]{2}{*}{$\begin{array}{l}314 \text { Textiles, } \\
\text { excepto prendas }\end{array}$} & & 0.3945 & 32.1886 & 15.4947 & 0.0001 & & 0.3945 & 525.0850 & $\begin{array}{ll}0 & 14.2646 \\
\end{array}$ & 0.0007 \\
\hline & Al menos $1 *$ & 0.1324 & 7.1036 & 3.8415 & 0.0077 & $1 *$ & 0.1324 & $4 \quad 7.1036$ & \begin{tabular}{l|l}
6 & 3.8415 \\
\end{tabular} & 0.0077 \\
\hline \multirow[t]{2}{*}{315 Prendas } & & 0.3540 & 22.0342 & 15.4947 & 0.0045 & & 0.4334 & 434.4854 & \begin{tabular}{l|l}
4 & 25.8721 \\
\end{tabular} & 0.0033 \\
\hline & Al men & 0.0038 & 0.1881 & 3.8415 & 0.6645 & * & 0.1144 & $\begin{array}{ll}4 & 6.0771 \\
\end{array}$ & \begin{tabular}{l|l}
12.5180 \\
\end{tabular} & 0.4505 \\
\hline \multirow[t]{2}{*}{316 Cuero y piel } & & 0.2588 & 21.8210 & 18.3977 & 0.0160 & & 0.2588 & \begin{tabular}{|l|l|} 
& 14.9757 \\
\end{tabular} & \begin{tabular}{l|l}
7 & 17.1477 \\
\end{tabular} & 0.1008 \\
\hline & Al menos $1^{*}$ & 0.1279 & 6.8453 & 3.8415 & 0.0089 & * & 79 & \begin{tabular}{|l}
9.8453 \\
9
\end{tabular} & \begin{tabular}{l|l}
3 & 3.8415 \\
\end{tabular} & .0089 \\
\hline \multirow[t]{2}{*}{321 Madera } & & 0.2819 & 16.7256 & 15.4947 & 0.0325 & & 0.2819 & 16.5575 & 514.2646 & 0.0213 \\
\hline & Al menos $1 *$ & 0.0034 & 0.1681 & 3.8415 & 0.6818 & $1 *$ & 0.0034 & $\begin{array}{ll}4 & 0.1681 \\
\end{array}$ & \begin{tabular}{l|l}
1 & 3.8415 \\
\end{tabular} & 0.6818 \\
\hline \multirow[t]{2}{*}{322 Papel } & & 0.3116 & 19.2258 & 15.4947 & 0.0130 & & 0.3116 & 18.6686 & \begin{tabular}{l|l}
6 & 14.2646 \\
\end{tabular} & 0.0094 \\
\hline & Al menos 1 * & 111 & 0.5571 & 3.8415 & 0.4554 & s 1 * & & 10.5571 & \begin{tabular}{l|l}
1 & 3.8415 \\
\end{tabular} & .4554 \\
\hline \multirow[t]{2}{*}{323 Impresión } & & 0.4434 & 36.9158 & 25.8721 & 0.0014 & & 0.4434 & 429.2975 & \begin{tabular}{l|l}
5 & 19.3870 \\
\end{tabular} & .0013 \\
\hline & Al menos 1 * & 0.1413 & 7.6183 & 12.5180 & 0.2844 & Al menos 1 * & 0.1413 & \begin{tabular}{|l|l|}
3 & 7.6183 \\
\end{tabular} & \begin{tabular}{l|l}
3 & 12.5180
\end{tabular} & 0.2844 \\
\hline \multirow[t]{2}{*}{$\begin{array}{l}324 \text { Deriv. del } \\
\text { petróleo y carbón }\end{array}$} & & 0.3547 & 22.7440 & 15.4947 & 0.0034 & & 0.3547 & $7 \quad 21.9056$ & 614.2646 & 0.0026 \\
\hline & Al menos $1^{*}$ & 0.0166 & 0.8384 & 3.8415 & 0.3599 & $51 *$ & 0.0166 & \begin{tabular}{|l} 
\\
\end{tabular} & \begin{tabular}{l|l}
4 & 3.8415 \\
\end{tabular} & 0.3599 \\
\hline \multirow[t]{2}{*}{325 Química } & & 0.3220 & 20.1731 & 15.4947 & 0.0091 & & 0.3220 & 19.4295 & \begin{tabular}{l|l}
5 & 14.2646 \\
\end{tabular} & 0.0070 \\
\hline & Al menos $1 *$ & 0.0148 & 0.7436 & 3.8415 & 0.3885 & Al menos $1 *$ & 0.0148 & \begin{tabular}{|l|l}
3 & 0.7436 \\
\end{tabular} & \begin{tabular}{l|l}
6 & 3.8415 \\
\end{tabular} & 0.3885 \\
\hline \multirow[t]{2}{*}{326 Plást. y hule } & & 0.4847 & 33.8306 & 15.4947 & 0.0000 & & 0.4847 & 333.1459 & \begin{tabular}{l|l}
9 & 14.2646 \\
\end{tabular} & 0.0000 \\
\hline & Al menos $1 *$ & 0.0136 & 0.6847 & 3.8415 & 0.4080 & $\operatorname{nos} 1 *$ & 0.0136 & 0.6847 & \begin{tabular}{l|l}
7 & 3.8415
\end{tabular} & 0.4080 \\
\hline \multirow[t]{2}{*}{$\begin{array}{l}327 \text { Prod. Min. no } \\
\text { met. }\end{array}$} & & 4878 & 35.3596 & 15.4947 & 0.0000 & & 0.4878 & \begin{tabular}{|l|l}
33.4521 \\
\end{tabular} & 114.2646 & 0.0000 \\
\hline & Al menos 1 * & 0.0374 & 1.9075 & 3.8415 & 0.1672 & s 1 * & 0.0374 & $4 \quad 1.9075$ & \begin{tabular}{l|l}
5 & 3.8415 \\
\end{tabular} & 0.1672 \\
\hline \multirow[t]{2}{*}{331 Met. básicas } & & 0.4245 & 29.4310 & 15.4947 & 0.0002 & & 0.4245 & 27.6282 & \begin{tabular}{l|l} 
& 14.2646
\end{tabular} & 0002 \\
\hline & Al menos 1 * & 0.0354 & 1.8027 & 3.8415 & 0.1794 & $\cos 1 *$ & 0.0354 & $4 \quad 1.8027$ & \begin{tabular}{l|l}
7 & 3.8415 \\
\end{tabular} & 0.1794 \\
\hline \multirow[t]{2}{*}{332 Prod. Met. } & & 0.4245 & 27.6282 & 14.2646 & 0.0002 & & 0.4334 & 28.4083 & \begin{tabular}{l|l}
3 & 19.3870
\end{tabular} & 0.0019 \\
\hline & Al menos 1 * & 0.0354 & 1.8027 & 3.8415 & 0.1794 & Al menos 1 * & 0.1144 & \begin{tabular}{|l|l|}
4 & 6.0771 \\
\end{tabular} & 112.5180 & 0.4505 \\
\hline \multirow[t]{2}{*}{333 Maq. y equipo } & & 0.3962 & 25.4979 & 15.4947 & 0.0011 & & 0.3962 & 25.2227 & \begin{tabular}{l|l}
7 & 14.2646
\end{tabular} & 0.0007 \\
\hline & Al menos $1 *$ & 0.0055 & 0.2753 & 3.8415 & 0.5998 & Al menos 1 * & 0.0055 & 0.2753 & \begin{tabular}{l|l}
3 & 3.8415 \\
\end{tabular} & 0.5998 \\
\hline \multirow[t]{2}{*}{$\begin{array}{l}334 \text { Comp. y } \\
\text { comun. }\end{array}$} & & 0.3793 & 28.9142 & 25.8721 & 0.0203 & & 0.3793 & 323.8483 & \begin{tabular}{l|l}
3 & 19.3870 \\
\end{tabular} & 0.0105 \\
\hline & Al menos $1 *$ & 0.0964 & 5.0659 & 12.5180 & 0.5869 & Al menos $1^{*}$ & 0.0964 & 5.0659 & $\begin{array}{ll}12.5180 \\
9\end{array}$ & 0.5869 \\
\hline $\begin{array}{l}335 \text { Equipo de gen. } \\
\text { Eléctrica. }\end{array}$ & & 0.3552 & 28.2331 & 25.8721 & 0.0250 & & 0.3552 & 21.9399 & \begin{tabular}{l|l}
9 & 19.3870 \\
\end{tabular} & 0.0208 \\
\hline & Al menos 1 * & 0.1183 & 6.2932 & 12.5180 & 0.4240 & Al menos 1 * & 0.1183 & \begin{tabular}{|l|l|} 
& 6.2932 \\
\end{tabular} & $\begin{array}{ll}2 & 12.5180 \\
\end{array}$ & 0.4240 \\
\hline 336 Transporte & & 0.3646 & 27.8964 & 25.8721 & 0.0276 & & 0.3646 & 22.6760 & 19.3870 & 0.0160 \\
\hline & Al menos $1 *$ & 0.0991 & 5.2204 & 12.5180 & 0.5651 & Al menos $1^{*}$ & 0.0991 & 5.2204 & \begin{tabular}{l|l}
4 & 12.5180
\end{tabular} & 0.5651 \\
\hline 337 Muebles & & 0.2918 & 18.1783 & 15.4947 & 0.0192 & & 0.2918 & \begin{tabular}{|l|}
17.2526 \\
\end{tabular} & \begin{tabular}{l|l}
6 & 14.2646 \\
\end{tabular} & 0.0164 \\
\hline & Al menos $1 *$ & 0.0183 & 0.9257 & 3.8415 & 0.3360 & Al menos 1 * & 0.0183 & $\begin{array}{ll} & 0.9257 \\
\end{array}$ & \begin{tabular}{l|l}
7 & 3.8415 \\
\end{tabular} & 0.3360 \\
\hline 339 Otras indus. & & 0.3468 & 21.3090 & 15.4947 & 0.0059 & & 0.3468 & 21.2940 & $\begin{array}{ll}0 & 14.2646\end{array}$ & 0.0033 \\
\hline & Al menos 1 * & 0.0003 & 0.0150 & 3.8415 & 0.9022 & Al menos 1 * & 0.0003 & \begin{tabular}{|l|l|} 
& 0.0150 \\
\end{tabular} & \begin{tabular}{l|l}
0 & 3.8415 \\
\end{tabular} & 0.9022 \\
\hline
\end{tabular}




\section{Cuadro 4}

\begin{tabular}{|c|c|c|c|c|}
\hline \multicolumn{5}{|c|}{$\begin{array}{c}\text { Características del componente cíclico de las manufacturas de EUA y de los subsectores } \\
\text { manufactureros de México }(2005-2009)\end{array}$} \\
\hline & $\begin{array}{l}\text { Desviación } \\
\text { estándar }\end{array}$ & Volatilidad $^{1}$ & Correlación $^{1}$ & 1 Autocorrelación \\
\hline Manufacturas de los EUA & 0.0146 & & & \\
\hline Manufacturas de México $^{2}$ & 0.0067 & 0.4573 & 0.8610 & \\
\hline 311 Industria alimentaria & 0.0061 & 0.4156 & 0.1092 & 0.5888 \\
\hline 312 Industria de las bebidas y del tabaco & 0.0088 & 0.6016 & 0.3536 & 0.6292 \\
\hline 313 Fabricación de insumos textiles & 0.0089 & 0.6116 & 0.2927 & 0.6452 \\
\hline $\begin{array}{l}314 \text { Confección de productos textiles, excepto } \\
\text { prendas de vestir }\end{array}$ & 0.0082 & 0.5629 & 0.2266 & 0.4004 \\
\hline 315 Fabricación de prendas de vestir & 0.0070 & 0.4764 & 0.2457 & 0.6782 \\
\hline $\begin{array}{l}316 \text { Fabricación de productos de cuero, piel y } \\
\text { materiales sucedáneos, excepto prendas de } \\
\text { vestir }\end{array}$ & 0.0079 & 0.5418 & 0.5353 & 0.4687 \\
\hline 321 Industria de la madera & 0.0179 & 1.2253 & 0.6386 & 0.7722 \\
\hline 322 Industria del papel & 0.0052 & 0.3554 & 0.6883 & 0.7434 \\
\hline 323 Impresión e industrias conexas & 0.0077 & 0.5289 & 0.3910 & 0.3567 \\
\hline $\begin{array}{l}324 \text { Fabricación de productos derivados del } \\
\text { petróleo y del carbón }\end{array}$ & 0.0187 & 1.2812 & 0.4428 & 0.1927 \\
\hline 325 Industria química & 0.0051 & 0.3517 & 0.3014 & 0.4638 \\
\hline 326 Industria del plástico y del hule & 0.0110 & 0.7545 & 0.9067 & 0.8612 \\
\hline $\begin{array}{l}327 \text { Fabricación de productos a base de } \\
\text { minerales no metálicos }\end{array}$ & 0.0137 & 0.9407 & 0.8521 & 0.8536 \\
\hline 331 Industrias metálicas básicas & 0.0088 & 0.6041 & 0.8493 & 0.8125 \\
\hline 332 Fabricación de productos metálicos & 0.0124 & 0.8463 & 0.8382 & 0.8367 \\
\hline 333 Fabricación de maquinaria y equipo & 0.0195 & 1.3362 & 0.7806 & 0.8931 \\
\hline $\begin{array}{l}334 \text { Fabricación de equipo de computación, } \\
\text { comunicación, medición y de otros equipos, } \\
\text { componentes y accesorios electrónicos }\end{array}$ & 0.0231 & 1.5821 & 0.4320 & 0.9153 \\
\hline $\begin{array}{l}335 \text { Fabricación de equipo de generación } \\
\text { eléctrica y aparatos y accesorios eléctricos }\end{array}$ & 0.0105 & 0.7185 & 0.6721 & 0.9153 \\
\hline 336 Fabricación de equipo de transporte & 0.0175 & 1.2014 & 0.8434 & 0.8515 \\
\hline $\begin{array}{l}337 \text { Fabricación de muebles y productos } \\
\text { relacionados }\end{array}$ & 0.0115 & 0.7849 & 0.7055 & 0.7549 \\
\hline 339 Otras industrias manufactureras & 0.0082 & 0.5628 & -0.0064 & 0.7264 \\
\hline
\end{tabular}




\section{Cuadro 5}

\begin{tabular}{|c|c|c|c|c|}
\hline \multicolumn{5}{|c|}{$\begin{array}{c}\text { Características del componente cíclico de las manufacturas de EUA y de los } \\
\text { estados de México }(2003 / 01-2009 / 03)\end{array}$} \\
\hline & $\begin{array}{l}\text { Desviación } \\
\text { estándar }\end{array}$ & Volatilidad $^{1}$ & Correlación ${ }^{1}$ & Aurocorrelación \\
\hline EUA & 0.0147 & & & 0.7738 \\
\hline Aguascalientes & 0.0670 & 4.5630 & 0.3670 & 0.1008 \\
\hline Baja California & 0.0398 & 2.7065 & 0.5152 & 0.3509 \\
\hline Baja California Sur & 0.0407 & 2.7726 & 0.1236 & 0.2307 \\
\hline Campeche (3) & 0.0397 & 2.7018 & 0.0771 & 0.1643 \\
\hline $\begin{array}{l}\text { Coahuila de } \\
\text { Zaragoza }\end{array}$ & 0.0683 & 4.6500 & 0.4755 & 0.3855 \\
\hline Colima & 0.0273 & 1.8600 & 0.3755 & 0.5389 \\
\hline Chiapas & 0.0918 & 6.2480 & 0.7183 & 0.6812 \\
\hline Chihuahua & 0.0951 & 6.4708 & 0.2223 & 0.0292 \\
\hline Distrito Federal (2) & 0.0297 & 2.0185 & 0.2389 & 0.1448 \\
\hline \begin{tabular}{|l|} 
Durango \\
\end{tabular} & 0.0308 & 2.0968 & 0.4007 & 0.0348 \\
\hline Guanajuato (2) & 0.0540 & 3.6778 & 0.1504 & 0.0590 \\
\hline Guerrero & 0.0258 & 1.7546 & 0.2454 & 0.3532 \\
\hline Hidalgo & 0.0266 & 1.8089 & 0.3958 & 0.3717 \\
\hline Jalisco (2) & 0.0380 & 2.5892 & 0.0528 & 0.3185 \\
\hline México (2) & 0.0277 & 1.8873 & 0.4081 & 0.0627 \\
\hline $\begin{array}{l}\text { Michoacán de } \\
\text { Ocampo }\end{array}$ & 0.0592 & 4.0300 & 0.5409 & 0.3950 \\
\hline Morelos & 0.0444 & 3.0198 & 0.0492 & 0.1912 \\
\hline Nayarit & 0.0520 & 3.5387 & -0.0137 & 0.5123 \\
\hline Nuevo León (2) & 0.0393 & 2.6787 & 0.5548 & 0.3583 \\
\hline Oaxaca & 0.0385 & 2.6222 & 0.0593 & 0.1033 \\
\hline Puebla & 0.0774 & 5.2658 & 0.3322 & 0.2222 \\
\hline Querétaro & 0.0361 & 2.4563 & 0.5357 & 0.1568 \\
\hline Quintana Roo & 0.0208 & 1.4177 & -0.0202 & 0.4220 \\
\hline San Luis Potosí & 0.0243 & 1.6532 & 0.1153 & 0.1239 \\
\hline Sinaloa & 0.0320 & 2.1807 & 0.3586 & 0.2983 \\
\hline \begin{tabular}{|l|} 
Sonora \\
\end{tabular} & 0.0738 & 5.0232 & 0.3414 & 0.3159 \\
\hline Tabasco & 0.0628 & 4.2752 & 0.1414 & 0.4885 \\
\hline Tamaulipas & 0.0321 & 2.1864 & 0.4766 & 0.2760 \\
\hline Tlaxcala & 0.0383 & 2.6096 & 0.1695 & 0.4756 \\
\hline \begin{tabular}{|l|} 
Veracruz de \\
Ignacio de la Llave
\end{tabular} & 0.0533 & 3.6274 & -0.0906 & 0.4331 \\
\hline Yucatán & 0.0376 & 2.5618 & 0.0522 & 0.3115 \\
\hline Zacatecas & 0.0361 & 2.4554 & 0.3077 & 0.2184 \\
\hline
\end{tabular}

Fuente: Estimaciones propias con datos de BIE de INEGI y del U.S. Department of Commerce: Bureau of Economic Analysis. 1. Respecto al índice de manufacturas de EUA. 2. Los números en paréntesis se refieren a los meses de rezago en el efecto de la autocorrelación. 


\section{Cuadro 6}

\begin{tabular}{|c|c|c|c|c|c|c|}
\hline $\begin{array}{l}\text { Los cinco subsectores de la } \\
\text { caídas en sus tasas de cre }\end{array}$ & $\begin{array}{l}\text { ustria } \\
\text { iento } \\
\text { SC }\end{array}$ & $\begin{array}{l}\text { manı } \\
\text { anual } \\
\text { CIAN }\end{array}$ & re 2 & $\begin{array}{l}\operatorname{mex} \\
\text { y } 20\end{array}$ & de & $\begin{array}{l}\text { mayores } \\
\text { lerdo al }\end{array}$ \\
\hline Periodo & 2005 & 2006 & 2007 & 2008 & 2009 & \begin{tabular}{|c|} 
Acumulado \\
$2008-$ \\
$2009^{1}$ \\
\end{tabular} \\
\hline $\begin{array}{l}336 \text { Fabricación de equipo de } \\
\text { transporte }\end{array}$ & $5.64 \%$ & $4.38 \%$ & $0.09 \%$ & $-13.64 \%$ & $-6.77 \%$ & $-20.41 \%$ \\
\hline $\begin{array}{l}334 \text { Fabricación de equipo de } \\
\text { computación, comunicación, } \\
\text { medición y de otros equipos, } \\
\text { componentes y accesorios } \\
\text { electrónicos }\end{array}$ & $0.54 \%$ & $-0.73 \%$ & $4.87 \%$ & $-7.84 \%$ & $-4.67 \%$ & $-12.52 \%$ \\
\hline $\begin{array}{l}337 \text { Fabricación de muebles y } \\
\text { productos relacionados }\end{array}$ & $3.78 \%$ & $1.50 \%$ & $-2.81 \%$ & $-8.90 \%$ & $-2.65 \%$ & $-11.55 \%$ \\
\hline $\begin{array}{l}314 \text { Confección de productos } \\
\text { textiles, excepto prendas de } \\
\text { vestir }\end{array}$ & $0.10 \%$ & $1.43 \%$ & $-4.20 \%$ & $-8.80 \%$ & $-0.99 \%$ & $-9.79 \%$ \\
\hline $\begin{array}{l}313 \text { Fabricación de insumos } \\
\text { textiles }\end{array}$ & $1.49 \%$ & $-1.74 \%$ & $-6.85 \%$ & $-7.92 \%$ & $-1.25 \%$ & $-9.17 \%$ \\
\hline $\begin{array}{l}315 \text { Fabricación de prendas de } \\
\text { vestir }\end{array}$ & $3.92 \%$ & $0.55 \%$ & $-1.57 \%$ & $-7.22 \%$ & $-1.95 \%$ & $-9.16 \%$ \\
\hline
\end{tabular}

Fuente: Estimaciones propias con datos del INEGI. Encuesta Industrial Mensual (EIM). 1. Hasta abril de 2009. 
Jorge Eduardo Mendoza Cota

Cuadro 7

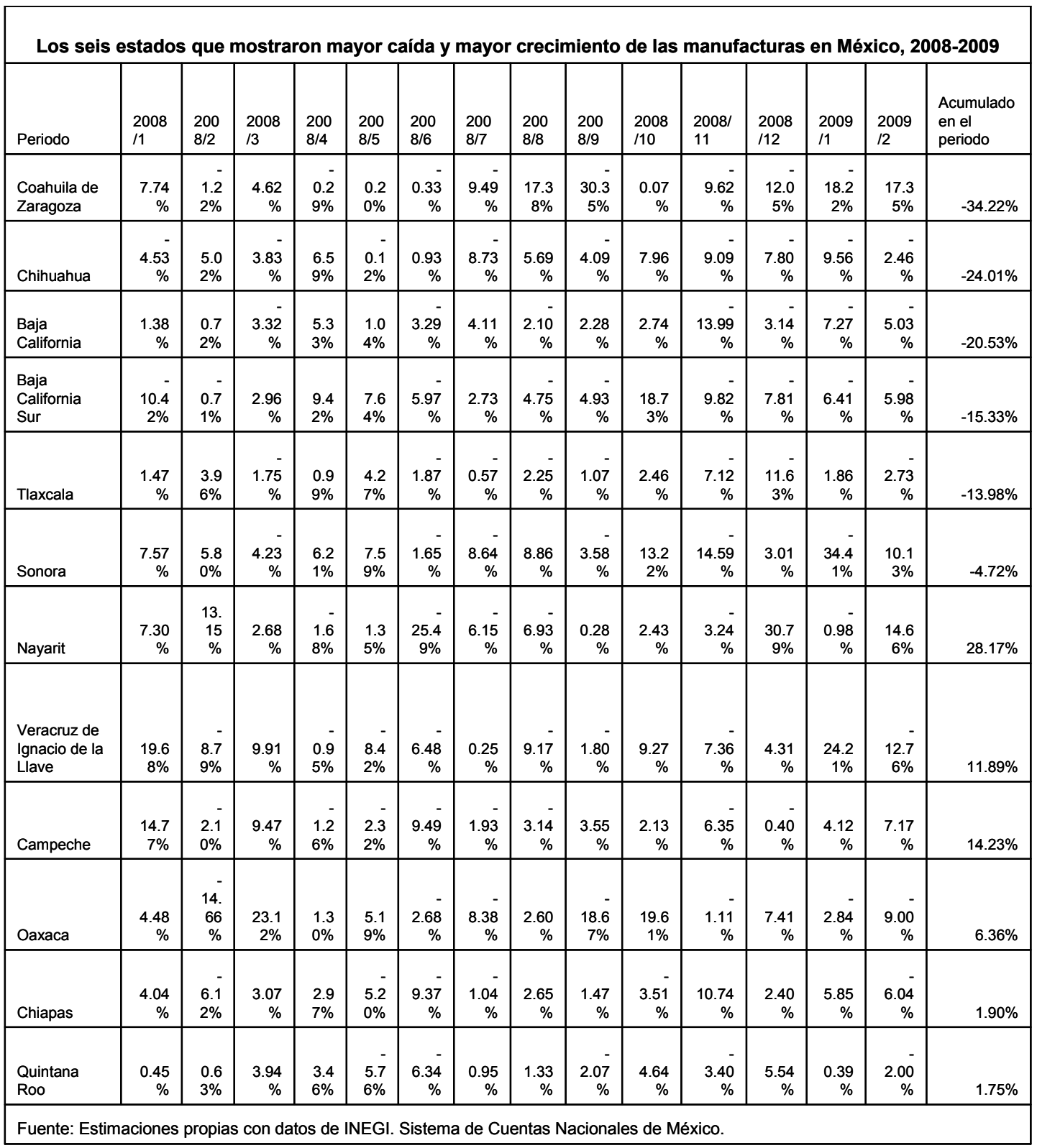




\section{Gráfica 1}

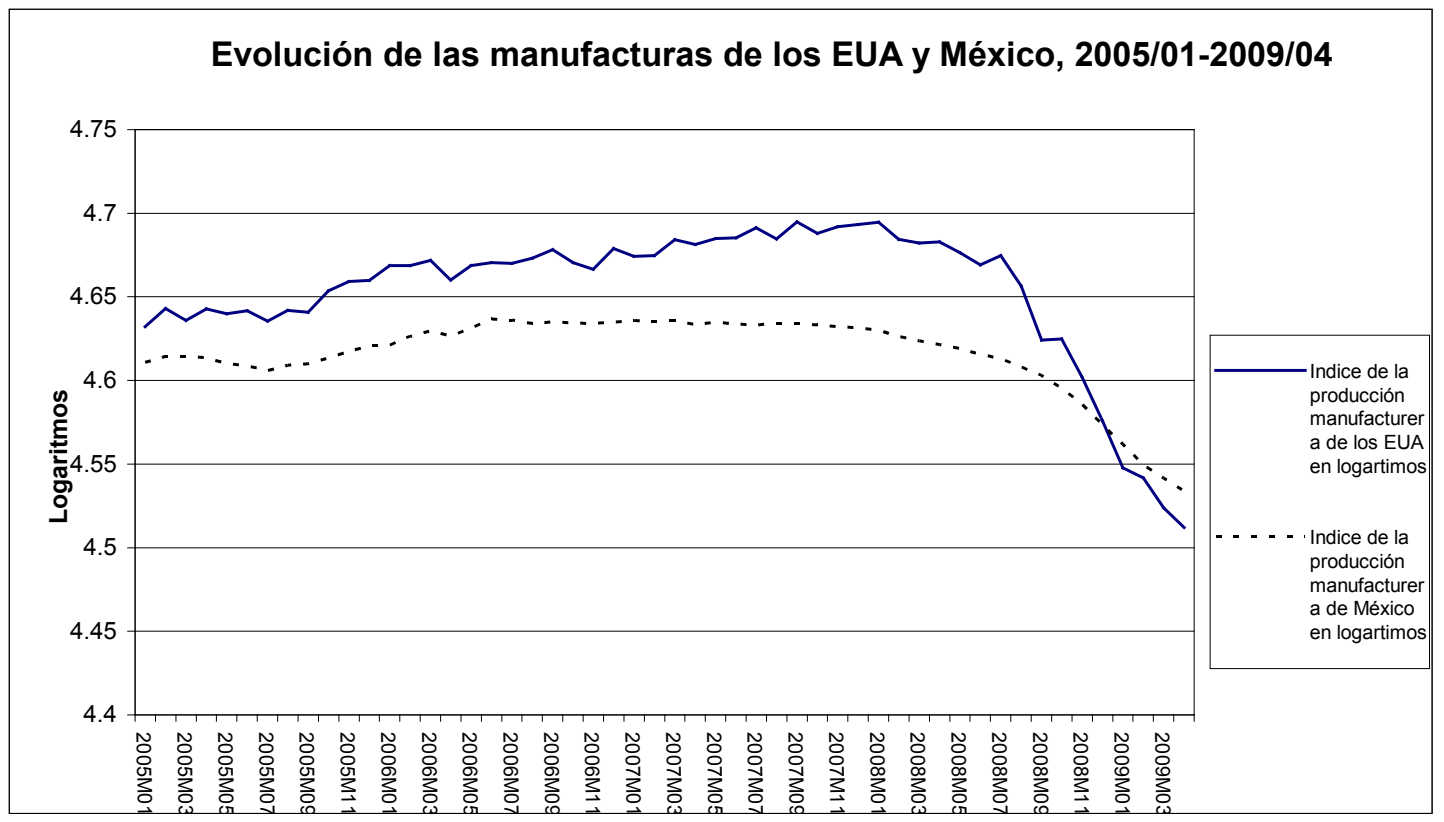

